similar survival outcomes to first-line LT and might be an appropriate treatment strategy for some patients with HCC.

Del Gaudio et al. compared the efficacy and safety of $L R$ and salvage $L T$ versus $L T$ in patients with cirrhosis and HCC who were suitable candidates for LT. First-line LT was performed in 147 patients, whereas 80 patients with preserved hepatic function underwent potentially curative LR. HCC recurred in 39 of the patients in the LR group and salvage LT was performed in 16 (10 for tumor recurrence and 6 for hepatic decompensation), whereas the remainder were considered untreatable by LT.

No significant difference in 5-year overall survival was found between patients who underwent LR (with or without salvage LT) and those who underwent LT (66\% vs $73 \%)$, although 5 -year disease-free survival was lower in the LR group ( $41 \%$ vs $71 \% ; P=0.001)$. Operative mortality, tumor recurrence, 5 year overall and disease-free survival were all similar in LR patients who underwent salvage LT and those who underwent first-line LT

The authors conclude that LR in patients with cirrhosis and HCC who are suitable candidates for transplant, with salvage LT in cases of recurrence or liver decompensation, is a safe and effective alternative to LT.

Original article Del Gaudio M et al. (2008) Liver transplantation for recurrent hepatocellular carcinoma on cirrhosis after liver resection: University of Bologna experience. Am J Transplant 8: 1177-1185

\section{Transgastric peritoneoscopy and liver biopsy is feasible in humans}

Many demonstrations of natural orifice transluminal endoscopic surgery have been performed in animal models; a US study has now demonstrated the feasibility of transgastric endoscopic examination of the peritoneum in humans. Transgastric liver biopsy was also performed.

Steele et al. recruited three patients with morbid obesity (mean BMl $40 \mathrm{~kg} / \mathrm{m}^{2}$ ) who were scheduled to undergo laparoscopic gastric bypass surgery. After division of the stomach to create a $20 \mathrm{ml}$ gastric pouch, the gastric wall incision for subsequent gastrojejunostomy was used to provide endoscopic access to the peritoneum. A flexible, forward-viewing endoscope was advanced into the peritoneal cavity and a liver biopsy performed using standard endoscopic forceps. Biopsy samples were taken from liver segments II, III and IVb for histologic grading of steatohepatitis. Systematic examination of the liver, epigastric area, small intestine and omentum was carried out without laparoscopic assistance. An endoscopic light source was used, but illumination was found to be adequate even when the light was switched off. The peritoneal exploration and liver biopsy took $2.1 \mathrm{~min}, 4.3 \mathrm{~min}$ and $6.0 \mathrm{~min}$ to perform in the three patients. There were no complications or signs of infection in the month following the procedure.

The authors conclude that flexible transgastric peritoneoscopy is feasible, simple to perform, and could prove a useful complement to laparoscopic abdominal surgery, particularly in morbidly obese patients. Further studies to optimize transgastric entry and gastric closure techniques for stand-alone natural orifice transluminal endoscopic surgical procedures are planned.

Original article Steele K et al. (2008) Flexible transgastric peritoneoscopy and liver biopsy: a feasibility study in human beings (with videos). Gastrointest Endosc 68: 61-66

\section{Cardiac dysfunction during liver transplantation is common and not predicted by tests}

Patients with cirrhosis can be affected by cardiomyopathy, which is normally masked by the low systemic vascular resistance characteristic of liver disease. Cardiomyopathy, therefore, only manifests in situations of increased cardiovascular stress. Liver transplantation induces considerable cardiac stress, and poor cardiac function in this setting could worsen surgical and perioperative outcomes.

Ripoll et al. retrospectively analyzed data from 209 patients with cirrhosis who had undergone liver transplantation. Clinical, echocardiographic, hepatic and hemodynamic baseline data were compared with data collected during transplantation $10 \mathrm{~min}$ after graft reperfusion - the time of maximum cardiac stress. Abnormal cardiac response was defined as a decrease in left ventricle stroke work despite an increase in filling pressures.

A total of $47(22.5 \%)$ patients had an abnormal cardiac response during liver transplantation $10 \mathrm{~min}$ after reperfusion, despite the use of 